\title{
Feasibility Study of Carbon Nanotube Microneedles for Rapid Transdermal Drug Delivery
}

\author{
Bradley J. Lyon ${ }^{1}$, Adrianus I. Aria ${ }^{1}$, and Morteza Gharib ${ }^{1}$ \\ ${ }^{1}$ Graduate Aerospace Laboratories, California Institute of Technology, Pasadena, CA 91125, \\ U.S.A.
}

\begin{abstract}
We introduce a new approach for fabricating hollow microneedles using verticallyaligned carbon nanotubes (VA-CNTs) for rapid transdermal drug delivery. Here, we discuss the fabrication of the microneedles emphasizing the overall simplicity and flexibility of the method to allow for potential industrial application. By capitalizing on the nanoporosity of the CNT bundles, uncured polymer can be wicked into the needles ultimately creating a high strength composite of aligned nanotubes and polymer. Flow through the microneedles as well as in vitro penetration of the microneedles into swine skin is demonstrated. Furthermore, we present a trade study comparing the difficulty and complexity of the fabrication process of our CNT-polymer microneedles with other standard microneedle fabrication approaches.
\end{abstract}

\section{INTRODUCTION}

Microneedles are envisioned to provide a painless, self-administered alternative to standard hypodermic injection. Specifically, hollow microneedles allow for a delivery architecture that is more flexible than other microneedle designs such as solid microneedles, drug-coated microneedles, or dissolving microneedles. This is because hollow microneedles allow for variable delivery rate from rapid injection to emulate a hypodermic injection to slow steady delivery to mimic intravenous drug therapy. Additionally, since the hollow microneedle is designed to be inert with respect to the drug, many current drugs that are delivered into the skin can be directly used in the hollow microneedle architecture [1].

Previous studies on the fabrication of hollow microneedles have focused on top-down fabrication approaches using either silicon, metal, or glass. While these approaches have yielded functioning microneedles, their fabrication involves iterated etching or micromachining techniques that ultimately add complexity to the fabrication and limit industrial-scale application [1]. Here, we introduce a new perspective on forming hollow microneedles by fabricating microneedles using a bottom-up approach by using vertically-aligned carbon nanotubes (VACNTs). VA-CNTs are first used as a scaffold for forming the shape of the microneedle and then as a fiber component in a CNT-polymer composite to create a high strength material capable of penetrating the skin.

By utilizing standard methods to grow VA-CNTs, including catalyst patterning and thermal chemical vapor deposition (CVD), we directly produce a hollow microneedle. In designing the CNT-polymer composite microneedle, the final product must achieve four mechanical objectives: (i) have high mechanical strength under compression to achieve skin penetration, (ii) conformally coat the VA-CNTs with polymer allowing the microneedle to retain its original shape from catalyst patterning, (iii) anchor microneedles to a common polymer base for easy transfer from the growth substrate to drug delivery platforms, and (iv) maintain an unobstructed hollow cavity for drug delivery. 
These objectives are interdependent and thus careful choice and application of polymer must be applied to create a functioning microneedle. Here, we demonstrate our approach using the negative photoresist SU8-2025 (MicroChem, Newton, MA) as a candidate polymer. The high UV absorbance of CNTs allow us to selectively cure the microneedle device. Thus, the polymer base can be cured while leaving the polymer within the microneedle cavity uncured allowing for removal of the polymer in later steps. SU8-2025 is chosen due to its property of forming thick films through spin coating (typically $25 \mu \mathrm{m}$ at $3000 \mathrm{rpm}$ ) and high elastic modulus of $3 \mathrm{GPa}$ [2]. A previous study has shown that low viscous SU8, such as SU8-2002 (MicroChem, Newton, MA), can be spin coated on VA-CNTs to create a composite [3]. However, the thin film created by this resist through spin coating ( $2 \mu \mathrm{m}$ at $3000 \mathrm{rpm})$ is too fragile for making a structurally supportive base for the microneedles [2]. After proving that the CNT-polymer composite microneedle is mechanically feasible with SU8-2025, future studies will focus on broadening the number of polymers that can be employed in this technique.

\section{EXPERIMENT}

Vertically aligned CNTs are fabricated via thermal CVD on a silicon substrate patterned with catalyst in the shape of hollow circles which act as the microneedle template. To obtain this pattern, the silicon wafer is masked with photoresist patterned through photolithography into the desired microneedle geometry. Alumina and iron of thickness $10 \mathrm{~nm}$ and $1 \mathrm{~nm}$ respectively are then deposited on the wafer via electron beam deposition. After deposition, photoresist is stripped from the wafer to finalize the substrate preparation. For the CVD process, ethylene and hydrogen gas is flowed across the substrate at $750^{\circ} \mathrm{C}, 600$ torr and $490 \mathrm{sccm}$ and $210 \mathrm{sccm}$ respectively for an hour to achieve VA-CNT growth.

At the end of CVD, the VA-CNTs are patterned into hollow cylinders matching the catalyst pattern of $150 \mu \mathrm{m}$ outer diameter and inner cavity diameter of $25 \mu \mathrm{m}$ (Figure 1, Step 1). The height of the microneedles can be varied from $150 \mu \mathrm{m}$ to $400 \mu \mathrm{m}$. Patterned VA-CNTs alone cannot act as a microneedle. VA-CNTs are prone to buckling and have an exceedingly low modulus under compression of only $550 \mathrm{kPa}$ [4]. Additionally, the VA-CNTs must be connected to a common base other than the growth substrate to allow for the microneedles to be transferred onto a device platform for drug delivery.

Starting with the patterned VA-CNTs, we incorporate SU8-2025 to create the composite microneedle. SU8-2025 is dropcasted onto the VA-CNT sample which is then passively wicked into the interspacing of the VA-CNTs. Next, the sample is spin coated at $3000 \mathrm{rpm}$ for 60 seconds to remove the excess material and simultaneously create a base that is thick enough (nominal $25 \mu \mathrm{m}$ ) to allow for the microneedles to be easily removed from the silicon substrate in later steps with minimal damage to the microneedles and the polymer base (Step 2). However, the high viscosity of the SU8-2025 causes the resist to pool in the inner cavity of the microneedle. After spin coating, the sample is soft baked at $95^{\circ} \mathrm{C}$ for four minutes.

The photoresist is selectively cured under oblique incidence UV light (UV/Visible Light Exposure Chamber, MTI Corp., Richmond, CA) at $30 \mathrm{~mW} / \mathrm{cm}^{2}$ for up to 1 minute (Step 3). In this arrangement, the polymer base is cured along with the SU8 embedded within the VA-CNT interspacing. The SU8 in the inner cavity remains uncured due to the oblique incidence of the UV light preventing direct exposure of the inner cavity. Additionally, the VA-CNTs in the CNTSU8 composite attenuate the amount of UV light reaching the inner cavity through the composite. Following UV exposure, the sample goes through a post exposure bake 


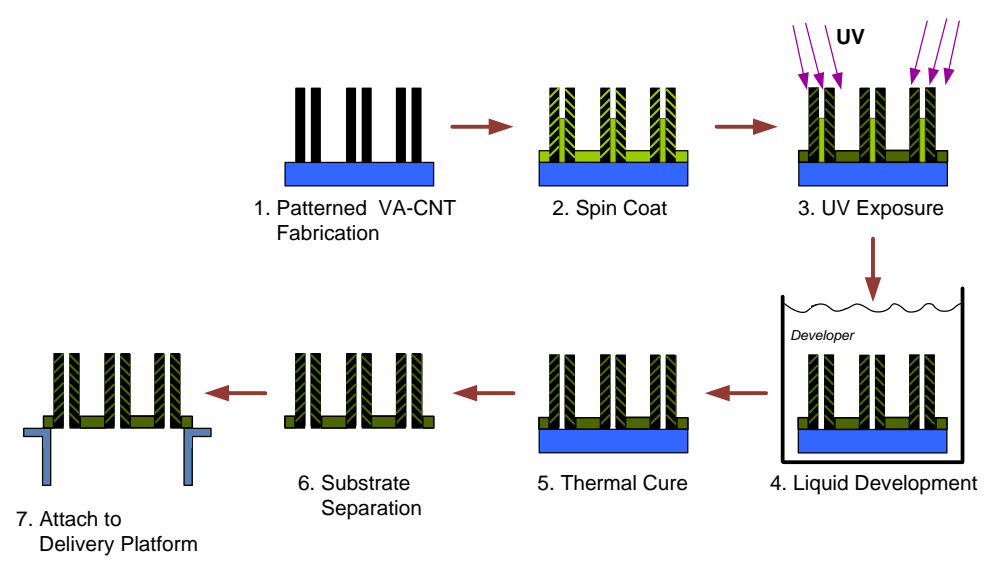

Figure 1- Microneedle fabrication process starting with CVD fabrication of patterned VA-CNTs.

for 3 minutes at $95^{\circ} \mathrm{C}$. The microneedles are then submerged in SU8 Developer (MicroChem, Newton, MA) to clear the uncured photoresist from the inner cavity (Step 4). The submerged sample is placed on a shaker table set to $150 \mathrm{rpm}$ for 10 minutes. Following development, the sample is rinsed in isopropanol and cured in a vacuum oven at $150{ }^{\circ} \mathrm{C}$ for 20 minutes (Step 5).

At this point, the CNT-SU8 composite microneedle is fully formed with a clear inner cavity and a common SU8 base. By taking advantage of the poor adhesion between SU8 and the silicon substrate as well as the thick SU8 base layer, the microneedles can be removed mechanically with a razor blade or tweezers (Step 6). The device is then transferred onto a delivery platform that connects the inner cavity of the microneedles to a liquid reservoir for drug flow (Step 7).

\section{RESULTS \& DISCUSSION}

\section{CNT-SU8 Microneedle}

The CNT-SU8 microneedles are spaced on a $1 \mathrm{~mm}$ grid to ensure that each needle independently penetrates the skin (Figure $2 \mathrm{a}, \mathrm{b}$ ). Ideal microneedle height was found to be in the range of $200 \mu \mathrm{m}$ to $250 \mu \mathrm{m}$. Microneedles above this range are more susceptible to buckling failure. Microneedle heights below $200 \mu \mathrm{m}$ were found to be too short to achieve consistent skin penetration. SU8 conformally coats the microneedle allowing the final product to retain the original pattern defined by catalyst patterning and CVD growth of the VA-CNTs. Comparing the VA-CNT structure to the CNT-SU8 composite structure, we see that the SU8 fully envelops the interspacing between the nanotubes creating a single solid composite structure (Figure 2 c,d). SEM imaging of the underside of the needle confirms that the inner cavity of the needle is clear of polymer after fabrication (Figure 2e).

Liquid flow through the microneedle is demonstrated by connecting a microneedle array to a water filled syringe. Through hand actuation, the microneedle is capable of expelling water into the air at rates of up to $600 \mu \mathrm{L} / \mathrm{min}$ per needle (Figure 3a). Despite the relatively thin polymer base of approximately $25 \mu \mathrm{m}$, the base shows no signs of fatigue or cracking under high flow rates. Achieving flow greater than $100 \mu \mathrm{L} / \mathrm{min}$ per needle under minimal actuation pressure demonstrates the low hydraulic resistance of the device which ultimately lowers the work needed to flow liquid through the microneedles at any flow rate. For the current microneedle height, we anticipate for in vitro drug delivery using delivery rates of about 1 to $10 \mu \mathrm{L} / \mathrm{min}$ per needle and 
volumes of about $100 \mu \mathrm{L}$ due to the restrictive permeability of the skin. Longer microneedles penetrating deeper into the skin may enable higher flow rates and may lead to future applications of the microneedle as an auto-injector for rapid delivery of rescue medication.

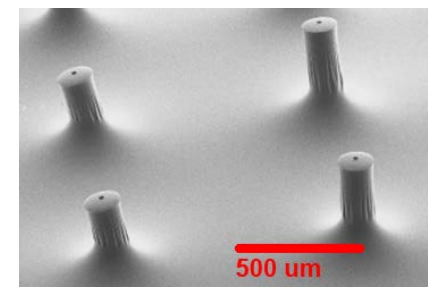

(a)

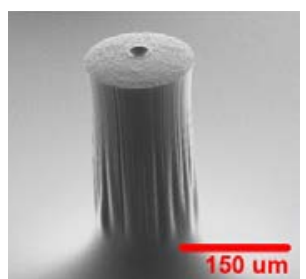

(b)

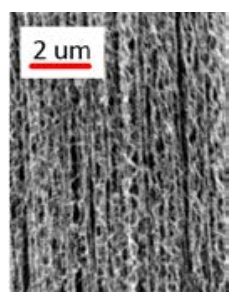

(c)

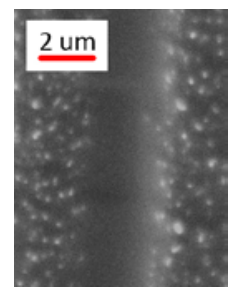

(d)

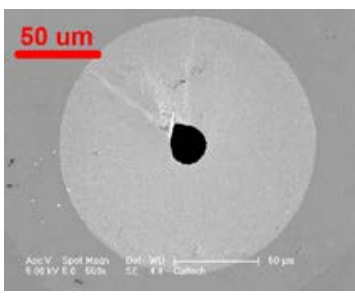

(e)

Figure 2- (a), (b) CNT-SU8 composite microneedle array. Outer diameter $150 \mu \mathrm{m}$ with $25 \mu \mathrm{m}$ inner diameter. Structure of microneedle (c) before and (d) after SU8 incorporation. (e) Underside of microneedle after fabrication showing the inner cavity is clear of polymer.

In vitro skin penetration is demonstrated on thin $(<0.5 \mathrm{~mm})$ samples of dorsal swine skin prepared by using a dermatome to cut full thickness skin samples. Microneedles were pressed by hand into the swine skin and achieved penetration at tip pressures in the range of 60MPa to $90 \mathrm{MPa}$. Prior to penetration, the microneedles are coated with dry methylene blue powder. Upon contact with the interstitial fluid in the skin, the methylene blue dye is passively released from the needle marking the point of contact with the skin (Figure 3b). The clear pattern of the 2 x 2 microneedle array indicates positive penetration and ultimately demonstrates that the CNT-SU8 composite has sufficient strength to achieve skin penetration. To present, all of the mechanical objectives of the microneedle outlined previously have been achieved. Ongoing work is now looking to build upon these results by characterizing the microneedle's in vitro liquid delivery into the skin as well as broadening the method to demonstrate incorporation of other polymers.

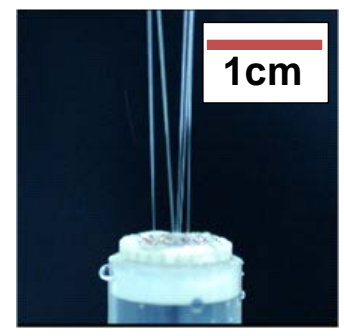

(a)

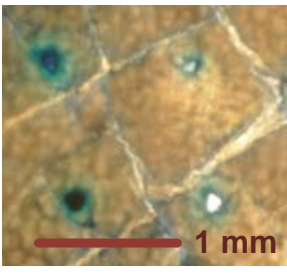

(b)

Figure 3- (a) Water jets from the microneedle array with exit velocity of about $600 \mu \mathrm{L} / \mathrm{min}$ per needle. (b) In vitro swine skin penetration marks by microneedles coated in methylene blue dye.

\section{Fabrication Method Comparison}

A trade study was conducted by comparing the laboratory difficulty in fabricating the CNT-SU8 composite microneedle to the current primary approaches for microneedle fabrication including silicon microneedles and micromolding. Fabrication of both silicon and CNT-SU8 microneedles offer similar advantages in terms of geometry customization and parallel processing of large numbers of microneedles. In characterizing the fabrication process for silicon microneedles, reactive ion etching (RIE) is presumed to be the primary method for defining the microneedle shape with additional processes such as wet etching or micromachining used as 
secondary methods to optimize the microneedle geometry[1, 5-7]. For each process used in the fabrication method, a difficulty factor between 1 through 3 is assigned based on the difficulty of the process on the laboratory scale (Table I).

Table I: Trade study of fabrication difficulty for CNT-SU8 composite microneedles and silicon microneedles.

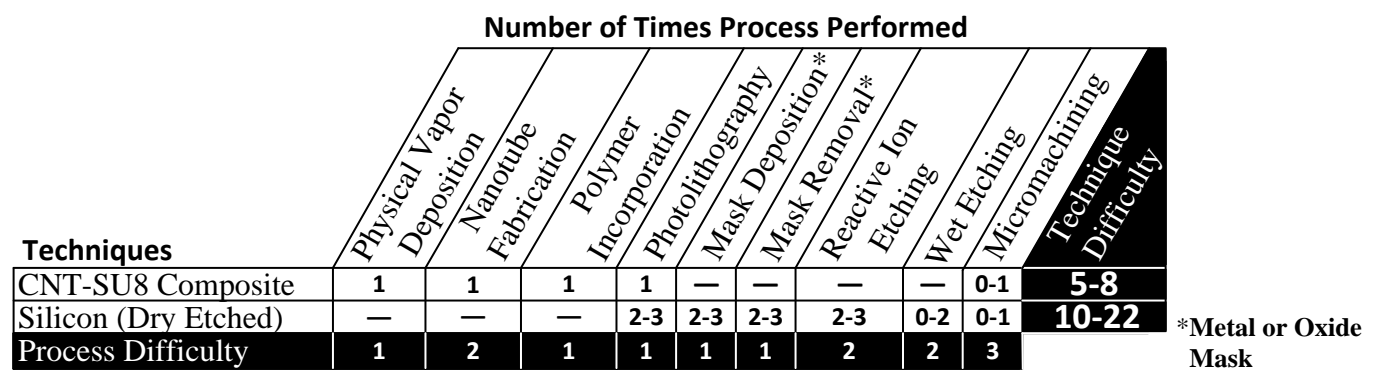

CNT-SU8 composite has a smaller total technique difficulty than silicon showing that the CNT-polymer method has the potential to be significantly simpler than the silicon approach. This is because creating a hollow cavity in the silicon approach requires iterative etching steps which in turn require iterative processing steps. In contrast, the entire geometry of the CNT-SU8 needle is defined from the sequential steps of catalyst patterning and nanotube fabrication. Another important consideration in the CNT-SU8 approach is that incorporation of SU8 on the VA-CNTs relies on a combination of simple and passive mechanisms such as capillary action and UV exposure that can be easily executed on both the laboratory and industrial level. The primary fabrication challenge for the CNT-polymer microneedle is the fabrication of VA-CNTs. However, the recent increase of commercial fabrication options should lower the overall fabrication difficulty in the long run.

To present, only a hollow cylinder geometry has been considered for our CNT-SU8 microneedle. In comparison with other microneedles, a tapered shape is typically preferred to minimize the tip area as the required penetration force scales linearly with tip area [8]. Previous work has demonstrated that drug release from the top of the microneedle is susceptible to skin occlusion which increases the hydraulic resistance of delivery. A suggested alternative is to release the drug from the side of the microneedle to optimize the delivery by lowering the hydraulic resistance $[1,5]$. The fabrication of CNT-SU8 microneedles can be amended to incorporate these more complex geometries if desired. The microneedles can be modified after polymer incorporation via micromachining or before polymer incorporation using techniques such as capillography [3] or focused ion beam milling. Further study of the in vitro drug release profile for our current microneedle is necessary before determining if the potential benefit of optimizing the shape of our microneedles outweighs the cost of increased fabrication difficulty.

Micromolding is another common approach to producing hollow microneedles by electroplating micromolds to produce a hollow metallic microneedle. Typical master structures for micromolding are fabricated by micromachining or laser drilling of a bulk material which can be time consuming and ultimately limits the minimum feature size of the needle [1,9]. However after the master structure is complete, the micromolding process allows for fast fabrication of large numbers of microneedles. Both the silicon and CNT-SU8 needle can achieve much finer feature size and thus may both be potentially used as a master structure for micromolding. A previous study has demonstrated the successful use of CNT-SU8 composites for general micromolding applications [3]. 


\section{CONCLUSIONS}

A new approach to fabricating hollow microneedles has been shown using verticallyaligned carbon nanotubes and SU8-2025. By taking advantage of self-assembly, VA-CNTs can be simply adapted as a hollow microneedle by incorporating SU8-2025. SU8-2025 allows for the creation of a strong composite with VA-CNTs while simultaneously creating a supportive base for the microneedle array. Initial experiments have shown that the CNT-SU8 needles can penetrate the skin in vitro and can structurally support high flow rates of up to $600 \mu \mathrm{L} / \mathrm{min}$ per needle. Further studies will investigate expanding the number of polymers that can be used in this architecture as well as in vitro characterization of the microneedle's delivery performance.

In comparing the fabrication of CNT-SU8 microneedles with other approaches, we find that the CNT-SU8 fabrication method is potentially simpler than that of silicon microneedles. CNT-SU8 composites may also be incorporated as master structures in micromolds to allow for feature size on the order of several microns. Laboratory scale fabrication represents only a single aspect for comparing microneedles. Future trade studies will need to take into account the performance, cost, and scalability aspects of the CNT-polymer microneedle to properly identify future applications and markets for this technology.

\section{ACKNOWLEDGMENTS}

We acknowledge Zcube s.r.l for their financial support of this work. We also acknowledge the Kavli Nanoscience Institute and the Geology and Planetary Sciences Analytical Facility for their support in running experiments.

\section{REFERENCES}

1. Y.-C. Kim, J.-H. Park and M. R. Prausnitz, Advanced Drug Delivery Reviews 64 (14), 1547-1568 (2012).

2. Microchem, SU-8 2000 Processing Guidelines.

3. M. De Volder, S. H. Tawfick, S. J. Park, D. Copic, Z. Zhao, W. Lu and A. J. Hart, Advanced materials 22 (39), 4384-4389 (2010).

4. L. Ci, J. Suhr, V. Pushparaj, X. Zhang and P. M. Ajayan, Nano letters 8 (9), 2762-2766 (2008).

5. H. J. G. E. Gardeniers, R. Luttge, E. J. W. Berenschot, M. J. De Boer, S. Y. Yeshurun, M. Hefetz, R. van't Oever and A. van den Berg, Microelectromechanical Systems, Journal of 12 (6), 855-862 (2003).

6. B. Ma, S. Liu, Z. Gan, G. Liu, X. Cai, H. Zhang and Z. Yang, Microfluidics and Nanofluidics 2 (5), 417-423 (2006).

7. L. M. Yu, F. E. H. Tay, D. G. Guo, L. Xu and K. L. Yap, Sensors and Actuators A: Physical 151 (1), 17-22 (2009).

8. S. P. Davis, B. J. Landis, Z. H. Adams, M. G. Allen and M. R. Prausnitz, Journal of biomechanics 37 (8), 1155-1163 (2004).

9. J. J. Norman, S. O. Choi, N. T. Tong, A. R. Aiyar, S. R. Patel, M. R. Prausnitz and M. G. Allen, Biomedical microdevices 15 (2), 203-210 (2013). 